\title{
Micro RNAS And Potential Role as Obesity Predictors At Period Of Covid-19
}

\author{
Carmine Finelli ${ }^{1,2 *}$ \\ ${ }^{1}$ Department of Internal Medicine, Ospedale Cav. R. Apicella - ASL Napoli 3 Sud, Via di Massa, Italy \\ ${ }^{2}$ Covid Hospital Boscotrecase - ASL Napoli 3 Sud, Via Lenza 3, Italy
}

*Corresponding author: Carmine Finelli, Department of Internal Medicine, Ospedale Cav. R. Apicella - ASL Napoli 3 Sud,

Via di Massa, 1, 80040 Pollena (Napoli), Covid Hospital Boscotrecase - ASL Napoli 3 Sud, Via Lenza 3, Italy

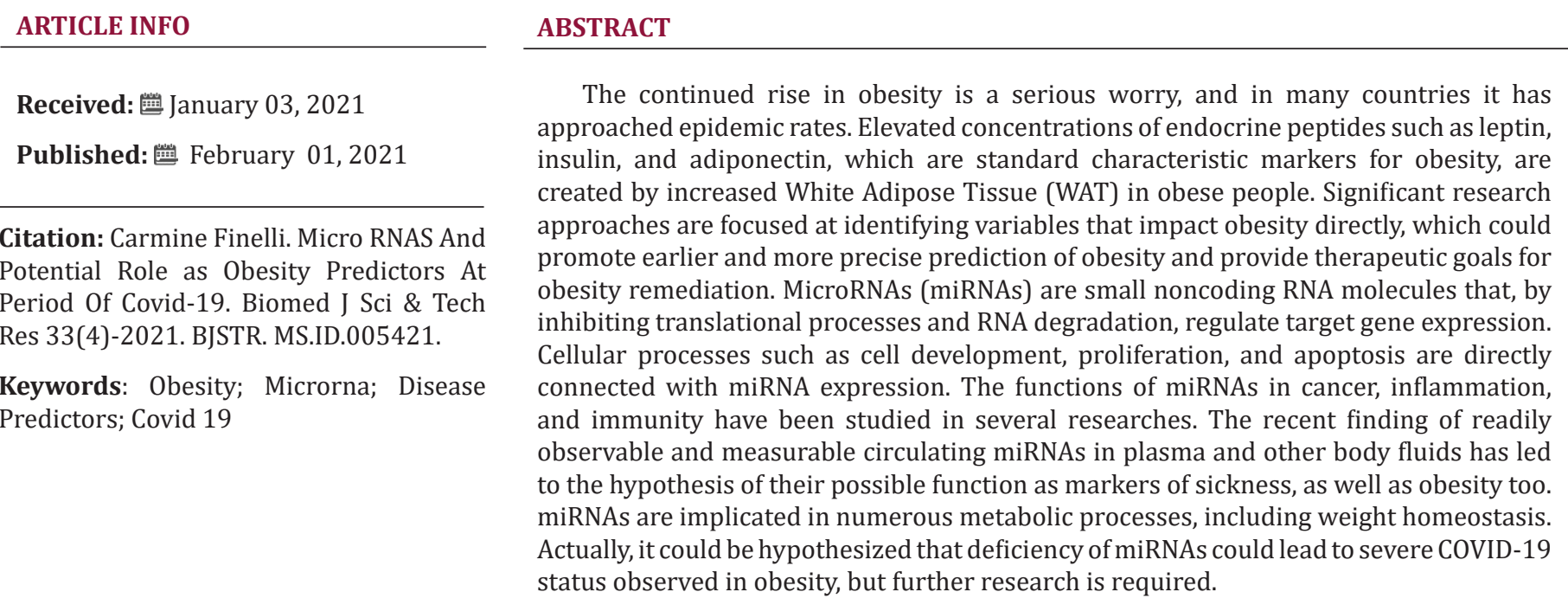

\section{Editorial}

The continued rise in obesity is a serious worry, and in many countries it has approached epidemic rates. Elevated concentrations of endocrine peptides such as leptin, insulin, and adiponectin, which are standard characteristic markers for obesity, are created by increased White Adipose Tissue (WAT) in obese people. Significant research approaches are focused at identifying variables that impact obesity directly, which could promote earlier and more precise prediction of obesity and provide therapeutic goals for obesity remediation [1]. This overall prevalence happens early in life as well. 110 million children and teenagers between the ages of 2-19 have obesity in general [1,2]. Obesity increases the susceptibility to an increased risk of complications in this demographic, with a greater number of cardio-respiratory, musculoskeletal and psychological disorders during puberty and adulthood, with an increased possibility of developing diabetes, impairment and even premature death $[3,4]$. The obesity-related complications are connected to the chronic inflammation in the adipose tissue that actually occurs in a low grade. In this tissue, its normal function is modified throughout obesity with changes in its cytokine development $[5,6]$.

Proteins such as IL-6, IL-8, IL-18, leptin, TNF- $\alpha$, CCL2, and resistin have been correlated with pro-inflammatory conditions and the progression of obesity-related complications, among others $[5,7,8]$. Setting up the pathway involved in the regulation of genes correlated with this immune response at the molecular level will allow an advancement in early-stage obesity treatment in order to minimize complications in adulthood. By binding to a complementary sequence of mRNA, molecules such as microRNAs (miRNAs) regulate gene expression $[6,9,10]$. Mechanisms that depend on miRNAs control up to $30 \%$ of all human genes $[6,11,12]$. Multiple pathways are affected by miRNAs, like insulin production, immune-mediated inflammation, adipokine expression, adipogenesis, lipid metabolism and food intake control $[6,10,11,13]$. Disruptions in the expression of miRNAs impair 
the expression of the gene and thus the cellular function in the pathological phase [14]. In the beginning phases, the introduction of innovative approaches to recognize the progress from obesity to inflammation will help to explain the various mechanisms that control this process. Even, for this population, it will provide details on proper management.

\section{MicroRNAs and Obesity}

MicroRNAs (miRNAs) are small noncoding RNA molecules that, by inhibiting translational processes and RNA degradation, regulate target gene expression [15]. Cellular processes such as cell development, proliferation, and apoptosis are directly connected with miRNA expression. The functions of miRNAs in cancer, inflammation, and immunity have been studied in several researches $[16,17]$. New researches have shown that miRNA profiles, including circulating miRNAs and those related to cancer or inflammatory process, can be defined from blood $[18,19]$. Circulating miRNAs are important markers for common diseases because they are relevant to human blood in a surprisingly stable state, and the findings may have a high degree of individual reproducibility $[1,20]$. Numerous miRNAs demonstrate typical dysregulation of obese patients in fat tissues and blood [21-23]. These research indicates that the circulating miRNA expression patterns represent the omental fat tissue expression patterns and act as obesity predictors. The importance of circulating miRNAs for obesity is still remain unclear, while research is trying to expose the connections between miRNAs and obesity. In terms of obesity, multiple researchers have described different miRNA expression profiles, and these findings are discussed in the literature [22,24,25]. Many research concentrate on model systems, so there is a lack of human subject knowledge. Obesity is a dynamic disorder and variations in miRNA expression profiles could be produced by intrinsic differences between fat tissues or individuals, such as adipocyte phenotype and obesity-related parameters [26].

Gender-specific variations can also lead to a change in miRNA expression profiles recorded in the literature [27]. The high circulating miR-122 expression levels increase the risk of obesity, which has also been correlated with higher anthropometric or biochemical parameters, such as body mass index, waist to height ratio, fat percentage, serum high-density lipid levels, triglycerides [28]. For a complete understanding of obesity-related miRNAs, these variables should be regarded. Infection with severe acute respiratory syndrome coronavirus 2 (SARS-CoV-2) can produce an abnormal host immune response and can lead to serious illness, a life-threatening disease that occurs more commonly in patients with comorbidities such as hypertension, diabetes, and obesity. The role of various human and SARS-CoV-2 miRNAs in the pathophysiology of COVID-19 has recently been shown in some studies [29]. Human corona virus infection is highly dependent on host miRNA involved in host innate immune response control and inflammation-modulatory miR-146a is among the first miRNAs induced by virus immune response [30].

\section{Discussion}

In conclusion, miRNAs are short non-coding RNA sequences synthesized in the nucleus of the cell via a complex multi-stage biosynthetic process beginning with RNA polymerase II; more than 2500 mature miRNAs are identified in the human genome. A broad range of biological processes, such as cell differentiation, proliferation and development, cell-to-cell communication, cell metabolism and apoptosis, are regulated by miRNAs. Insulin signalling, immune-mediated inflammation, adipokine expression, adipogenesis, lipid metabolism, and food intake appear to also be regulated by miRNAs. There is suggestion that miRNAs might also have a role in molecular processes linked to cellular pathways of certain diseases, such as viral infections, cancer, diabetes, obesity and cardiovascular disease. The recent finding of readily observable and measurable circulating miRNAs in plasma and other body fluids has led to the hypothesis of their possible function as markers of sickness, as well as obesity too. miRNAs are implicated in numerous metabolic processes, including weight homeostasis. Actually, it could be hypothesized that deficiency of miRNAs could lead to severe COVID-19 status observed in obesity, but further research is required.

\section{Disclosure Statement}

The authors declare that there are no conflicts of interest.

\section{References}

1. Lin H, Tas E, Børsheim E, Mercer KE (2020) Circulating miRNA Signatures Associated with Insulin Resistance in Adolescents with Obesity. Diabetes Metab Syndr Obes 13: 4929-4939.

2. Boonmanunt S, Pattanaprateep O, Ongphiphadhanakul B, Mc Kay G, Attia J, et al. (2020) Evaluation of the effectiveness of behavioural economic incentive programmes for the promotion of a healthy diet and physical activity: a protocol for a systematic review and network meta-analysis. BMJ Open 10(12): e046035.

3. Cotter EW, Hornack SE, Fotang JP, Pettit E, Mirza NM (2020) A pilot openlabel feasibility trial examining an adjunctive mindfulness intervention for adolescents with obesity. Pilot Feasibility Stud 6: 79.

4. Correa-Burrows P, Blanco E, Gahagan S, Burrows R (2020) Validity assessment of the single-point insulin sensitivity estimator (spise) for diagnosis of cardiometabolic risk in post-pubertal hispanic adolescents. Sci Rep 10(1): 14399.

5. Finelli C (2020) Obesity and immunotherapy: the surprisingly positive association! Immunotherapy 12(8): 541-544.

6. Filardi T, Sabato C, Lubrano C, Santangelo C, Morano S, et al. (2020) MicroRNA Modulation by Dietary Supplements in Obesity. Biomedicines 8(12): 545.

7. Finelli C, Tarantino G (2013) What is the role of adiponectin in obesity related non-alcoholic fatty liver disease? World J Gastroenterol 19(6): 802-812.

8. Selvaraju V, Babu JR, Geetha T (2019) Association of salivary C-reactive protein with the obesity measures and markers in children. Diabetes Metab Syndr Obes 12: 1239-1247.

9. Annese T, Tamma R, De Giorgis M, Ribatti D (2020) microRNAs Biogenesis, Functions and Role in Tumor Angiogenesis. Front Oncol 10: 581007.

10. Chen L, Huang K, Yi K, Huang Y, Tian X, et al. (2020) Premature MicroRNABased Therapeutic: A “One-Two Punch" against Cancers. Cancers (Basel) 12(12): 3831. 
11. Huai Y, Zhang W, Chen Z, Zhao F, Wang W, et al. (2020) A Comprehensive Analysis of MicroRNAs in Human Osteoporosis. Front Endocrinol (Lausanne) 11: 516213.

12. Assmann TS, Cuevas-Sierra A, Riezu-Boj JI, Milagro FI, Martínez JA (2020) Comprehensive Analysis Reveals Novel Interactions between Circulating MicroRNAs and Gut Microbiota Composition in Human Obesity. Int J Mol Sci 21(24): 9509.

13. Chakraborty C, Sharma AR, Sharma G, Lee SS (2020) Therapeutic advances of miRNAs: A preclinical and clinical update. J Adv Res 28: 127 138.

14. Kalayinia S, Arjmand F, Maleki M, Malakootian M, Singh CP (2021) MicroRNAs: roles in cardiovascular development and disease. Cardiovasc Pathol 50: 107296

15. Stavast CJ, Erkeland SJ (2019) The Non-Canonical Aspects of MicroRNAs: Many Roads to Gene Regulation. Cells 8(11): 1465.

16. Nelson MC, O'Connell RM (2020) MicroRNAs: At the Interface of Metabolic Pathways and Inflammatory Responses by Macrophages. Front Immunol 11: 1797.

17. Heyn GS, Corrêa LH, Magalhães KG (2020) The Impact of Adipose Tissue-Derived miRNAs in Metabolic Syndrome, Obesity, and Cancer Front Endocrinol (Lausanne) 11: 563816.

18. Kotańska M, Szafarz M, Mika K, Dziubina A, Bednarski M, et al. (2020) PSB 603 - a known selective adenosine A2B receptor antagonist - has anti-inflammatory activity in mice. Biomed Pharmacother 135: 111164

19. Najafi S, Ghanavat M, Shahrabi S, Gatavizadeh Z, Saki N (2021) The effect of inflammatory factors and their inhibitors on the hematopoietic stem cells fate. Cell Biol Int.

20. Condrat CE, Thompson DC, Barbu MG, Bugnar OL. Boboc A, et al. (2020) miRNAs as Biomarkers in Disease: Latest Findings Regarding Their Role in Diagnosis and Prognosis. Cells 9(2): 276.

21. Landrier JF, Derghal A, Mounien L (2019) MicroRNAs in Obesity and Related Metabolic Disorders. Cells 8(8): 859.

\section{ISSN: 2574-1241}

DOI: 10.26717/BJSTR.2021.33.005421

\section{Carmine Finelli. Biomed J Sci \& Tech Res}

This work is licensed under Creative Commons Attribution 4.0 License

Submission Link: https://biomedres.us/submit-manuscript.php
22. Gharanei S, Shabir K, Brown JE, Weickert MO, Barber TM, et al. (2020) Regulatory microRNAs in Brown, Brite and White Adipose Tissue. Cells 9(11): 2489.

23. Tait S, Baldassarre A, Masotti A, Calura E, martini P, et al. (2020) Integrated Transcriptome Analysis of Human Visceral Adipocytes Unravels Dysregulated microRNA-Long Non-coding RNA-mRNA Networks in Obesity and Colorectal Cancer. Front Oncol 10: 1089.

24. Thibonnier M, Esau C, Ghosh S, Wargent E, Stocker C (2020) Metabolic and energetic benefits of microRNA-22 inhibition. BMJ Open Diabetes Res Care 8(1): e001478.

25. Gao Y, Yuan X, Zhu Z, Wang D, Liu Q, et al. (2020) Research and prospect of peptides for use in obesity treatment (Review). Exp Ther Med 20(6): 234.

26. Pheiffer C, Willmer T, Dias S, Abrahams Y, Louw J, et al. (2020) Ethnic and Adipose Depot Specific Associations Between DNA Methylation and Metabolic Risk. Front Genet 11: 967.

27. Tsamou M, Vrijens K, Wang C, Winckelmans E, Neven KY, et al. (2020) Genome-wide microRNA expression analysis in human placenta reveals sex-specific patterns: an ENVIRONAGE birth cohort study. Epigenetics 6: 1-16.

28. González-Arce LM, Lara-Riegos JC, Pérez-Mendoza GJ, Rubi-Castellanos R, Vega-Marcin M, et al. (2020) High expression levels of circulating microRNA-122 and microRNA-222 are associated with obesity in children with Mayan ethnicity. Am J Hum Biol 23: e23540.

29. Abedi F, Rezaee R, Hayes AW, Nasiripour S, Karimi G (2020) MicroRNAs and SARS-CoV-2 life cycle, pathogenesis, and mutations: biomarkers or therapeutic agents? Cell Cycle 31: 1-11.

30. Roganović J (2020) Downregulation of microRNA-146a in diabetes, obesity and hypertension may contribute to severe COVID-19. Med Hypotheses 146: 110448.

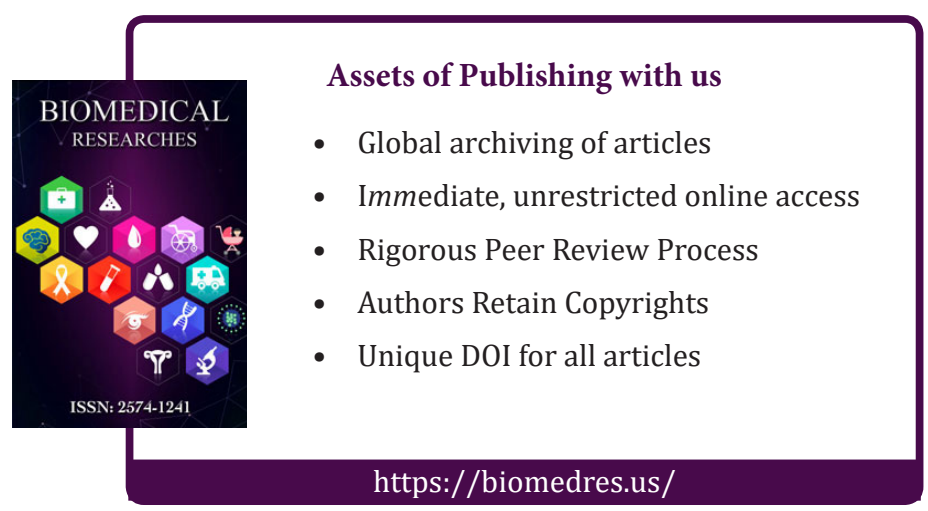

